\title{
Performance Analysis of Unsupervised Probabilistic, Possibilistic \& Plausibilistic Clustering Algorithms for Brain Tissue Segmentation
}

\author{
Ghanshyam D. Parmar \\ DA-IICT \\ Gandhinagar \\ India
}

\author{
Suman K. Mitra \\ DA-IICT \\ Gandhinagar \\ India
}

\begin{abstract}
Segmentation of brain tissues is one important process prior to many analysis and visualization tasks for magnetic resonance (MR) images. Clustering is one of the unsupervised techniques for doing the segmentation. Clustering is done with probabilistic, possibilistic and plausibilistic approaches. Most of segmentation techniques have relied on multi channel characteristics of MR images while a few studies have reported segmentation from single channel MR images. Owing to operator performance, limitation of equipment and environmental conditions MR images contain noise. This noise can lead to serious inaccuracies in the segmentation result. We conduct the research in measuring the performance of crisp and fuzzy clustering algorithms with probabilistic, possibilistic and plausibilistic approach in different noise level for single channel MR image. To validate the accuracy and robustness of the result of clustering algorithms we carried out experiments on simulated MR brain scans. The performance of algorithms is analyzed form three measures namely: number of iterations required, misclassification error and per class (tissue) misclassification error in different noise level present in the single-channel MR image.
\end{abstract}

\section{General Terms}

Pattern Recognition, Bio-Medical Image Analysis, Artificial Intelligence.

\section{Keywords}

Single-channel MR image, segmentation, unsupervised clustering algorithm, brain tissue classification.

\section{INTRODUCTION}

Magnetic resonance imaging (MRI) or nuclear magnetic resonance imaging (NMRI) $[1,2]$ is primarily medical imaging technique used in radiology to visualize internal structure of the body. MRI provides much greater contrast between different soft tissues of body. This ability makes it useful for neurological, musculoskeletal, cardio-vascular and oncological imaging [3]. Brain matter can be generally categorized as White Matter (WM), Gray Matter (GM) and Cerebrospinal Fluid (CSF) [4, 5]. Most of brain structures are anatomically defined by the boundaries of these tissue classes [4-6]. So we need a method of segmenting tissues in classes. It is an important step for quantitative analysis of the brain and its anatomical structures.
Brain tissue classification is also important step for detection of various pathological conditions affecting brain parenchyma [79]. It is also used for surgical planning and simulation [10] and three dimensional visualization for diagnosis and detection of abnormalities [11, 12]. It is also useful in the study of brain development $[13,14]$ and human aging $[15,16]$.

In MR imaging, images are produced based on intensities achieved by three tissue characteristics namely: $\mathrm{T} 1$ relaxation time, $\mathrm{T} 2$ relaxation time and proton density (PD). The images obtained by these properties are known as T1- weighted MR images, T2-weighted MR images and proton density MR images respectively. The effect of these parameters on image can be varied based on the adjusting the parameters like time to echo (TE) and time to repeat of the pulse sequence [17]. By using different parameters or number of echoes in the pulse sequence, a multitude of nearly registered images with different characteristics of same object can be achieved. If only a single MR image of the object is available such an image is referred to as single-channel (single-echo) image, and in case when numbers of MR images of the same object at same section are obtained, they are referred as multichannel (multi-spectral or multi-echo) images [18]. For a given scanning time, the voxel sizes achieved in multi-spectral images are larger than those achieved with single-channel images. This ability of finer voxel sizes makes single-channel images more suitable for precise and accurate quantitative measurements of anatomical structures and tissues. But multi-channel image provide more information at given voxel size than single-channel $[17,18]$.

Most of segmentation techniques have relied on multi-spectral characteristics of MR images while a few studies have reported segmentation form single-channel MR images [19].

Clustering is the unsupervised process of classification of objects, based on similarities among them. It is unsupervised technique as we do not have any prior information about the class of the data point or object. The backbone of clustering is to expose the hidden structure for the purpose of classification or data modeling. Clustering is done by making the partition among the data points. The aim of clustering on data is to make partition among the data points which have dissimilar characteristics and data points in the same group have similar characteristics. The partition to make the cluster can be crisp (hard) or fuzzy (soft). The approach to make the partition can be probabilistic, possibilistic or plausibilistic. These there approaches with their 
hard and soft variants are described in Section 2. It deals with algorithms based on probabilistic, possibilistic and plausibilistic approaches for unsupervised clustering techniques. The validation of the clustering results used in this work and the discussion of the results of these algorithms are described in the Section 3. Finally, the conclusion of our research is described in the Section 4.

\section{MATERIAL AND METHOD}

Given the data set of $\mathrm{n}$ points $X=\left\{x_{k} \mid k=1,2, \ldots, n\right\}$, where $x_{k}$ may be one dimensional or multi dimensional data point. In single-channel MR image each $x_{k}$ will be intensity either of T1 weighted or $\mathrm{T} 2$ weighted or PD weighted image and in case of multi channel MR image it will be combination of more than one from above three images. Each $x_{k}$ can also be features derived from these images.

In 1922 R. A. Fisher [20] introduced the method of Maximum Likelihood after the presentation of numerical procedure he presented in 1912 [21]. Maximum Likelihood is a popular statistical method for fitting statistical model to data and provides the estimate for parameters of the model [22]. Given a family of probability density function $f_{\theta}$, parameterized by an unknown parameter $\theta$ and n observations (data points) the Likelihood function can be defined by $L(\theta)=f_{\theta}\left(x_{1}, x_{2}, \ldots, x_{n}\right)$. The value of $\theta$ that maximizes $L(\theta)$ is the Maximum Likelihood estimate (MLE) of and is given by [22, 23] $\hat{\theta}=\arg \max L(\theta)$. In case when $f_{\theta}$ is assumed to be the weighted mixture of component probability density functions, the Maximum Likelihood estimate is given by [24]. The clustering algorithm based on Maximum Likelihood estimate is known as Maximum Likelihood clustering [24, 25].

Bezdek and Dunn [26] proposed distance norm based on Fuzzy Maximum Likelihood estimate (FMLE). Gath and Geva [27] presented a clustering algorithm based on Fuzzy Maximum Likelihood estimate for hyper ellipsoidal clusters and clusters with variable shape and size. The Fuzzy Maximum Likelihood estimate clustering employs the distance norm based on FMLE proposed by Bezdek and Dunn [26]. This distance norm is given by

$$
\begin{aligned}
D_{i k}\left(x_{k}, v_{i}\right) & =\frac{\sqrt{\operatorname{det}\left(F_{m i}\right)}}{\alpha_{i}} \exp \frac{\left(x_{k}-v_{i}\right)^{T} F_{m i}^{-1}\left(x_{k}-v_{i}\right)}{2}, \\
F_{m i} & =\frac{\sum_{k=1}^{n}\left(\mu_{i k}\right)^{m}\left(x_{k}-v_{i}\right)\left(x_{k}-v_{i}\right)^{T}}{\sum_{k=1}^{n}\left(\mu_{i k}\right)^{m}},(2)
\end{aligned}
$$

where $F_{m i}$ is the fuzzy covariance matrix of the $i^{\text {th }}$ cluster, $v_{i}$ is called cluster center or cluster prototype, $U=\left(\mu_{i k}\right)$ is the fuzzy partitioning matrix, $\mathrm{m}$ is the weighting exponent controls the 'fuzziness' of the resulting cluster and $\alpha_{i}$ is aprior probability of selecting the $\mathrm{i}^{\text {th }}$ cluster. The distance in Eq. (1) is used in the calculation of $\mathrm{P}\left(\mathrm{i}=\mathrm{x}_{\mathrm{k}}\right)$, the probability of selecting the $\mathrm{i}^{\text {th }}$ cluster given the $\mathrm{k}^{\text {th }}$ data point, is given by

$$
P\left(i / x_{k}\right)=\frac{\frac{1}{D_{i k}\left(x_{k}, v_{i}\right)}}{\sum_{i=1}^{c} D_{i k}\left(x_{k}, v_{i}\right)} .(3)
$$

By using Eq. (1) and (3) the clustering is performed based on the distance norm of Fuzzy Maximum Likelihood estimate.

In 1978 for dealing with uncertainty and an alternative to probability theory Lotfi Zadeh [28] introduced a mathematical theory called Possibility Theory [29] as an extension of Fuzzy sets [30]. Krishnapuram and Keller [31] cast the clustering problem into the framework of possibility theory by making the data partition as possibilistic partition. This is done by minimizing the objective function given by [31]

$$
J(T, V)=\sum_{i=1}^{c} \sum_{k=1}^{n} t_{i k}^{m} D_{i k}^{2}+\sum_{i=1}^{c} W_{i} \sum_{k=1}^{n}\left(1-t_{i k}\right)^{m}
$$

where and $D_{i k}$ is squared inner product distance norm, $T=\left(t_{i} k\right)$ is typicality or possibility matrix and $\mathrm{W}_{\mathrm{i}}$ is cluster weight. The membership values are interpreted as the degree of possibility of the points belonging to the classes. In possibilistic partition of data, the degree of possibility of the point belonging to the class is the compatibility of that point with that class prototype or center. Owing to the need of typicality (possibility) and membership, N. Pal and J. Bezdek [32] extend the basic idea of PCM to fuzzy possibilistic c-means (FPCM). It also solves coincident clusters problem of PCM. Fuzzy possibilistic c-means partition of data can be achieved by minimizing the objective function given by [32]

$$
J(T, U, V)=\sum_{i=1}^{c} \sum_{k=1}^{n}\left(u_{i k}^{m} t_{i k}^{m}\right) D_{i k}^{2} \cdot(5)
$$

Like probability and possibility theory, to deal with partial and unreliable information, Dampster [33] and Shafer [34] introduced the Theory of Belief functions, also referred as Evidence Theory. It is a theoretical framework for reasoning with partial and unreliable information using different models of reasoning under uncertainty including Smets's Transferable Belief Model [35]. Denœux and Masson used the Evidence Theory for the purpose of clustering. They proposed the clustering algorithm called Evidential Clustering (EVCLUS) [36]. The basic idea for EVCLUS is the more similar two objects; the more plausible it is that they belong to the same cluster. Here the clustering of data points is done based on the concept of basic belief assignment (bba), whereby a mass of belief is assign to each possible subset of classes. After assigning bba to each data point the plausibility for each two objects be-longing to same class is used to do the clustering. Let $\mathrm{M}=\left(\mathrm{m}_{1}, \mathrm{~m}_{2}, \ldots, \mathrm{m}_{\mathrm{c}}\right)$ be credal partition, set $\Omega=$ $\left(\omega_{1}, \omega_{2}, \ldots, \omega_{c}\right)$ of $c$ classes making the partition of data and $\mathrm{F}=$ $A_{1}, A_{1}, \ldots, A_{f}$ be the set of focal elements. EVCLUS minimizes an error function inspired from Sammon's [37] stress function given by 


$$
I(M, a, b)=\frac{1}{C} \sum_{x<x^{\prime}} \frac{\left(a K_{x x^{\prime}}+b-D_{x x^{\prime}}\right)^{2}}{D_{x x^{\prime}}}, \text { (6) }
$$

where $\mathrm{a}$ and $\mathrm{b}$ are coefficients and $\mathrm{C}$ is normalization constant. $\mathrm{D}_{\mathrm{xx}}$, is the dissimilarity between data point $\mathrm{x}$ and $\mathrm{x}, \mathrm{K}_{\mathrm{xx}}$, is the degree of conflict between $\mathrm{x}$ and $\mathrm{x}$. Denœux and Masson introduced a new clustering method Evidential c-means (ECM) [38] algorithm, which is an evidential version of the fuzzy cmeans algorithm. Here the partition of data points is achieved by minimizing the objective function given by

$$
J(M, V)=\sum_{i=1}^{n} \sum_{A_{k} \neq \phi}^{n} c_{i}^{\alpha} m_{i k}^{\beta} D_{i k}^{2}+\sum_{i=1}^{n} \delta^{2} m_{i \phi}^{\beta},(7)
$$

subject to,

$$
\sum_{k, A_{k} \neq \phi} m_{i k}+m_{i \phi}, i=1, \ldots, n,(8)
$$

where parameter $\delta$ controls the number of data points considered as outliers, $\beta$ controls the fuzziness of the resulting cluster. $\mathrm{c}^{\alpha}{ }_{\mathrm{i}}$ are additional weighting coefficients for penalizing the subset in $\Omega$ of high cardinality, $\alpha$ controls the degree of penalization.

\section{RESULT VALIDATION \& DISCUSSION}

Even though different scalar validity measures like Partition Coefficient (PC), Classification Entropy (CE), Partition Index (SC), Separation Index(S), Xie and Beni's Index (XB), Dunn's Index and many more [39] for the validation of the result of the clustering algorithms are available we use the following: As the interest in computer-aided, quantitative analysis of medical image data is growing, the need for validation of such techniques is also increased. For the solution of validation problem, Simulated Brain Database (SDB) is available [40]. The Simulated Brain Database contains a set of realistic MRI data volumes [41] produced by an MRI simulator [42]. This data set is used in our work to evaluate the performance of the tissue classification algorithms in a setting where the truth is known [43]. The detail about the noise used in our work for analysis is described in [40- 43].

Table 1. Number of iterations required for clustering in different noise level

\begin{tabular}{lcccccc} 
& \multicolumn{7}{c}{ Noise Level in \% } \\
\cline { 2 - 7 } Algorithm & $\mathbf{0}$ & $\mathbf{1}$ & $\mathbf{3}$ & $\mathbf{5}$ & $\mathbf{7}$ & $\mathbf{9}$ \\
\hline ML & 27 & 28 & 30 & 35 & 36 & 40 \\
FML & 29 & 32 & 36 & 40 & 46 & 52 \\
PCM & 11 & 11 & 18 & 32 & 45 & 52 \\
FPCM & 16 & 24 & 33 & 38 & 48 & 70 \\
EVCLUS & 269 & 285 & 290 & 322 & 357 & 378 \\
ECM & 241 & 269 & 287 & 312 & 337 & 359 \\
\hline
\end{tabular}

The results of these algorithms in different noise level for singlechannel MR image data set are described in this section. As these algorithms are iterative algorithms, the number of iterations required for the algorithm in different noise level is shown in Table 1. In Maximum Likelihood (ML) clustering algorithm as the noise level in the MR image is increase from $0 \%$ to $9 \%$, the number of iterations required to do the clustering are also increase from 27 to 40, causing increase in the processing time to make clusters. In case of Fuzzy Maximum Likelihood (FML) clustering algorithm, the number of iterations required are also increase to 29 to 52 , as the noise in the MR image increase from $0 \%$ to $9 \%$. Also the numbers of iterations required are higher than that requires for the ML clustering algorithm. In Possibilistic C-Means (PCM) clustering the number of iterations required are also increases as the noise level in the MR image increase. However the iterations are still fewer than that of ML and FML clustering algorithms. The number of iterations required in case of Fuzzy Possibilistic C-Means (FPCM) clustering algorithm are increase as the noise level in the MR image increase. They are higher than that required for PCM clustering algorithm. However in lower and moderate noise level they are fewer, and in higher noise level they are higher than ML and FML clustering algorithms. In case of Evidential clustering (EVCLUS), the numbers of iterations required are also increase as the noise level in the MR image increase. Also the required iterations are higher than that of all the previous algorithms. In Evidential C-Means (ECM) required iterations are increase as the noise level in MR image in-crease. Compare to EVCLUS fewer number of iterations are required by ECM clustering algorithm, however they are still higher than ML, FML, PCM, and FPCM clustering algorithms.

Table 2. Misclassification Error in different noise level in (\%)

\begin{tabular}{lcccccc} 
& \multicolumn{6}{c}{ Noise Level in \% } \\
\cline { 2 - 7 } Algorithm & $\mathbf{0}$ & $\mathbf{1}$ & $\mathbf{3}$ & $\mathbf{5}$ & $\mathbf{7}$ & $\mathbf{9}$ \\
\hline ML & 24.3320 & 10.1706 & 4.7270 & 5.4471 & 7.9650 & 12.8879 \\
FML & 21.4957 & 10.7092 & 4.4149 & 5.2194 & 8.4258 & 39.7725 \\
PCM & 3.4647 & 3.1408 & 3.7680 & 6.2781 & 11.5763 & 17.9696 \\
FPCM & 1.3077 & 1.5024 & 3.0136 & 5.0766 & 9.0209 & 13.7213 \\
EVCLUS & 1.2715 & 1.4945 & 3.0109 & 5.0587 & 9.0189 & 16.1827 \\
ECM & 1.2155 & 1.4400 & 2.9565 & 5.0031 & 8.9776 & 16.0828 \\
\hline
\end{tabular}

The misclassification error in $\%$ for different noise level in above discussed algorithms has shown in Table 2. In ML clustering algorithm the misclassification error is higher in lower and higher noise level, while in moderate noise level it is nearer to the noise present in the MR image. In lower noise level the misclassification error is less in FML than the ML clustering algorithm. However in higher noise level the misclassification error has increased than that of ML clustering algorithm. Such behavior in the misclassification error is due to the underline assumption of probability distribution in ML and FML clustering algorithms. In PCM clustering algorithm the misclassification error increases as the noise in MR image increase. This error is less than the error in FML in lower noise level, whereas in 
higher noise level this error is higher than FML clustering algorithm. In FPCM clustering algorithm the misclassification error increases as the noise present in the MR image increase. This error is less than error present in PCM clustering algorithm. Also in lower noise level this error is less than ML and FML clustering algorithms. In EVCLUS algorithm the misclassification error increases as the noise in the MR image increase. Also except the higher noise level this error is less than error present in the FPCM clustering algorithm. In case of ECM clustering algorithm the misclassification error increases as the noise present in the MR image increase. This error is less than error in EVCLUS. Also except the higher noise level this error is less than all the previous discussed algorithms.

Table 3. Per Class (Tissue) misclassification error in different noise level in $(\%)$

\begin{tabular}{|c|c|c|c|c|c|c|c|}
\hline \multirow[b]{2}{*}{ Noise } & \multirow[b]{2}{*}{ Class } & \multicolumn{6}{|c|}{ Algorithm } \\
\hline & & $\overline{M L}$ & FML & $\overline{\text { PCM }}$ & FPCM & EVCLUS & $\overline{\text { ECM }}$ \\
\hline \multirow{3}{*}{$0 \%$} & CSF & 13.1026 & 19.2960 & $\overline{0}$ & 1.4975 & 3.4358 & 3.3315 \\
\hline & GM & 0 & 0 & 6.0496 & 2.3122 & 1.3126 & 1.2946 \\
\hline & $\mathbf{W M}$ & 76.6317 & 59.1685 & 2.6821 & 0.6304 & 0.7982 & 0.7812 \\
\hline \multirow{3}{*}{$1 \%$} & CSF & 6.5036 & 8.9079 & 12.9032 & 1.4781 & .31587 & 3.1169 \\
\hline & GM & 0.2024 & 0.0777 & 0.0155 & 2.5331 & 1.6569 & 1.6104 \\
\hline & $\mathbf{W M}$ & 21.5853 & 22.6302 & 3.8483 & 0.8567 & 1.1291 & 1.0515 \\
\hline \multirow{3}{*}{$3 \%$} & CSF & 2.1777 & 2.1191 & 14.4416 & 2.4152 & 5.1728 & 5.0678 \\
\hline & GM & 2.7196 & 2.8842 & 0.9115 & 5.4351 & 4.2769 & 3.9838 \\
\hline & WM & 7.1079 & 6.3340 & 4.2160 & 1.7063 & 2.1528 & 2.0479 \\
\hline \multirow{3}{*}{$5 \%$} & CSF & 5.2058 & 5.1422 & 13.2986 & 4.4957 & 6.8923 & 6.7568 \\
\hline & GM & 6.2811 & 7.2229 & 2.6737 & 8.6765 & 6.7893 & 6.5636 \\
\hline & WM & 5.5120 & 4.4356 & 8.4274 & 3.3469 & 4.1883 & 4.1353 \\
\hline \multirow{3}{*}{$7 \%$} & CSF & 7.4847 & 6.1175 & 4.6125 & 4.6125 & 8.35821 & 8.4830 \\
\hline & GM & 13.0980 & 19.3332 & 14.6975 & 14.6975 & 9.9289 & 9.8406 \\
\hline & WM & 6.0347 & 3.7682 & 7.8438 & 7.8438 & 10.5213 & 10.1392 \\
\hline \multirow{3}{*}{$9 \%$} & CSF & 13.5466 & 48.9286 & 4.1181 & 0 & 10.6928 & 10.5368 \\
\hline & GM & 21.9981 & All & 29.6461 & All & 15.7879 & 15.6177 \\
\hline & WM & 10.5659 & 0 & 20.3271 & 0.0383 & 24.5019 & 23.4500 \\
\hline
\end{tabular}

The most interesting part of the result is the misclassification error per class (tissue). As we are classifying the MR image in to Gray Matter (GM), White Matter (WM) and Cerebrospinal Fluid (CSF), we compute the misclassification error per class (tissue) for single-channel MR image after the clustering done by the discussed clustering algorithms. The per class misclassification error is shown in Table 3. As for the clustering algorithms in noise level of $0 \%$ to $9 \%$, Table 3 shows the misclassification error in GM, WM and CSF. As increase the noise level the per class misclassification error in all the discussed clustering algorithms increase. Aldo GM tissue has higher misclassification than that of WM and CSF tissues. In probabilistic clustering algorithms ML and FML, in lower noise level less misclassification in GM tissue is achieved but in the same it has shown increase in the misclassification in WM and CSF tissues, and in higher noise level although they have shown less misclassification in CSF and WM tissues the error in GM has also increased. FML has shown less per tissue misclassification error than ML clustering algorithm.

In possibilistic clustering algorithms PCM and FPCM, increase in noise level cause increase the misclassification error in GM tissue, also FPCM has shown less per class misclassification error than PCM clustering algorithm. In evidential clustering algorithms EVCLUS and ECM, the increase in the noise level causes increase in misclassification error in GM, WM and CSF tissues. ECM has shown less per tissue misclassification error than EVCLUS algorithm. In given noise level for all tissues ECM has shown less per class misclassification error than discussed algorithms.

The images of segmentation result are shown in Figure 1, where blue color denotes Gray Matter (GM), green color denotes White Matter (WM) and red color denotes CerebroSpinal Fluid (CSF).

Figure 1. Segmentation result in different noise level with discussed unsupervised clustering algorithms

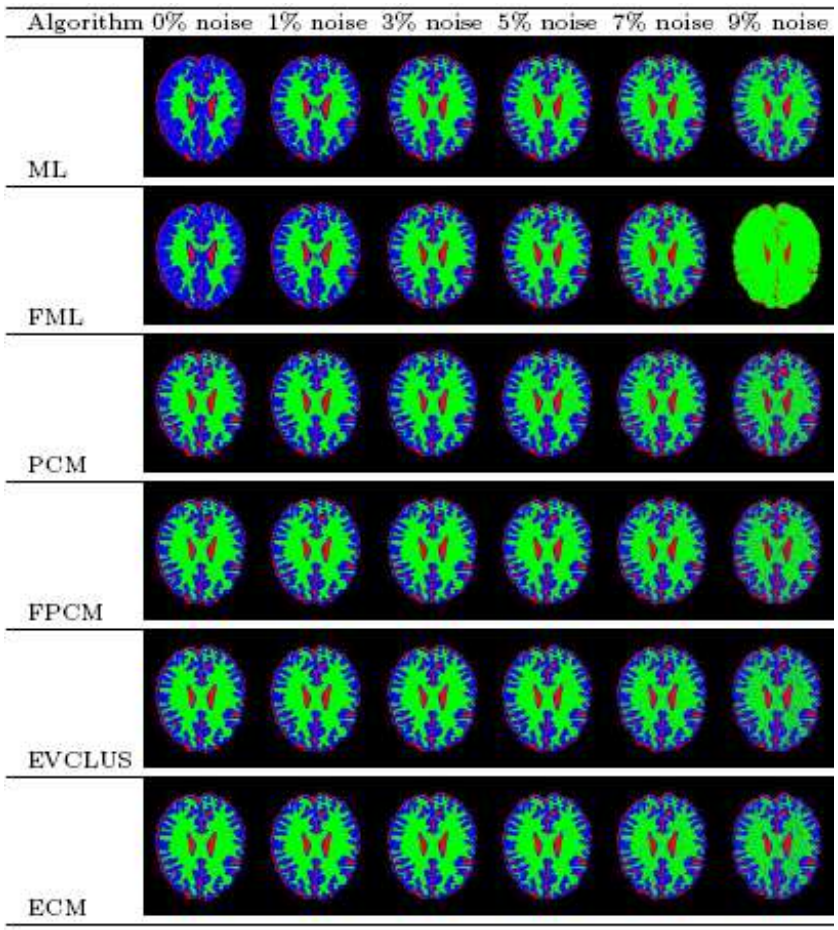

\section{CONCLUSION}

This paper presented a comparison of hard and fuzzy clustering algorithms with probabilistic, possibilistic and plausibilistic approach for tissue segmentation of single-channel MR image in different noise level. The performance of these algorithms has measured in different noise level using two measures namely: number of iterations required and the misclassification error. We 
successfully classify the brain tissues in single-channel MR image in different noise level using probabilistic, possibilistic and plausibilistic clustering algorithms. The effect of noise present in the single-channel MR image is measured on the number of iterations required to do the clustering, the misclassification error and the per class (tissue) misclassification error. For discussed algorithms as the noise level in the image increases the number of iterations required to do the clustering is also increases than that required with no noise condition. Also the misclassification error increases as the noise level in the MR image increases. The segmentation result improves as we move from probabilistic approach to possibilistic approach to plausibilistic approach with cost of number of iterations hence processing time required to do the clustering. In all these three approaches the soft clustering algorithms gave better segmentation result than that of the hard or crisp clustering algorithms for the same approach.

\section{REFERENCES}

[1] P. C. Lauterbur, Image formation by induced local interactions: Examples employing nuclear magnetic resonance, Nature 242 (5394) (1973) 190-191.

[2] P. C. Lauterbur, Magnetic resonance zeugmatography, Pure Appl. Chem. 40 (1-2) (1974) 149-157.

[3] L. F. Squire, Fundamentals of radiology, 4th Edition, Harvard University Press, 1988

[4] H. Damasio, Human brain anatomy in computerized images, 2nd Edition, Oxford University Press US, 2005.

[5] P. B. Henri M. Duvernoy, The human brain: surface, threedimensional sectional anatomy with MRI, and blood supply, 2nd Edition, Springer, 1999.

[6] J. Nolte, The human brain: an introduction to its functional anatomy, Mosby, 1981.

[7] C. R. Jack, Brain and cerebrospinal fluid volume: Measurement with mr imaging, Radiol. 178) (1991) 22\{24.

[8] T. E. Schlaepfer, G. J. Harris, A. Y. Tien, L. W. Peng, S. Lee, B. Federman, G. A. Chase, P. E. Barta, G. D. Pearlson, Decreased regional cortical gray matter volume in schizophrenia, American Journal of Psychiatry 151 (6) (1994) 842-848.

[9] W. Oslen, F. Longo, D. Mills, D. Norman, White matter disease in aids: Finding at MR imaging, Neuroradiol 169 (1988) 445-448.

[10] K. Fitzgerald, Medical electronics, IEEE Spectrum 28 (1) (1991) 76-78.

[11] R. Robb, Three-Dimensional Biomedical Imaging, New York: VCH, 1995.

[12] M. Brummer, R. Mersereau, R. Eisner, R. Lewine, Automatic detection of brain contours in MRI data sets, IEEE Trans. Med. Imag. 12 (2) (1993) 153-166.

[13] A. K. H. Miller, R. L. Alston, J. A. N. Corsellis, Variation with age in the volumes of gray and white matter in the cerebral hemispheres of man: Measurement with an image analyzer, Neuropathol., Appl. Neurobiol. 6 (1980) 119-132.

[14] T. Autti, R. Raininko, S. Vanhanen, M. Kallio, Santavuori, MRI of normal brain from early childhood to middle age, Neuroradiol 36 (1994) 644-648.

[15] D. G. M. Murphy, C. DeCarli, M. B. Schapiro, S. Rapoport, Horwitz, Age-related differences in volumes of subcortical nuclei, brain matter, and cerebrospinal fluid in healthy men as measured with magnetic resonance imaging, Arch. Neurol. 49 (1992) 839-845.

[16] K. O. Lim, R. B. Zipursky, M. C. Watts, A. Pfefferbaum, Decreased gray matter in normal aging: An in vivo magnetic resonance study, J. Gerontol.: Biolog. Sci. 47 (1) (1992) B26-30.

[17] R. H. Hashemi, W. G. Bradley, C. J. Lisanti, MRI: the basics, 2nd Edition, Lippincott Williams \& Wilkins, 2004.

[18] D. W. McRobbie, E. A. Moore, M. J. Graves, MRI from picture to proton, Cambridge University Press, 2003.

[19] J. Rajapakse, J. Giedd, J. Rapoport, Statistical approach to segmentation of single-channel cerebral MR images, IEEE Transactions on Medical Imaging 16 (2) (1997) 176-186.

[20] R. Fisher, On an absolute criterion for ftting frequency curves, Statistical Science 12 (1) (1997) 39-41.

[21] J. Aldrich, RA Fisher and the making of maximum likelihood 1912-1922, Statistical Science 12 (3) (1997) 162 176.

[22] A. Edwards, Likelihood, Cambridge Univ Pr, 1984.

[23] R. Royall, Statistical evidence: a likelihood paradigm, CRC Press, 1997.

[24] A. Dempster, N. Laird, D. Rubin, et al., Maximum likelihood from incomplete data via the EM algorithm, Journal of the Royal Statistical Society. Series B (Methodological) 39 (1) (1977) 1-38.

[25] R. Redner, H. Walker, Mixture densities, maximum likelihood and the EM algorithm, SIAM review 26 (2) (1984) 195-239.

[26] J. C. Bezdek, J. C. Dunn, Optimal fuzzy partitions: A heuristic for estimating the parameters in a mixture of normal distributions, IEEE Trans. Comput. 24 (8) (1975) 835-838.

[27] I. Gath, A. Geva, Unsupervised optimal fuzzy clustering, IEEE Transactions on Pattern Analysis and Machine Intelligence 11 (7) (1989) 773-780.

[28] L. Zadeh, Fuzzy sets as a basis for a theory of possibility, Fuzzy sets and systems 100 (1999) 9-34.

[29] L. Zadeh, A theory of approximate reasoning, Machine intelligence 9 (1) (1979) 149-194.

[30] L. Zadeh, Fuzzy sets*, Information and control 8 (3) (1965) 338-353.

[31] R. Krishnapuram, J. Keller, A possibilistic approach to clustering, Fuzzy Systems, IEEE Transactions on 1 (2) (1993) 98-110. 
[32] N. Pal, K. Pal, J. Bezdek, A mixed c-means clustering model, in: Fuzzy Systems, 1997., Proceedings of the Sixth IEEE Inter-national Conference on, Vol. 1, (1997), pp. 1121.

[33] A. Dempster, Upper and lower probabilities induced by a multi-valued mapping, The Annals of Mathematical Statistics 38 (2) (1967) 325-339.

[34] G. Shafer, A mathematical theory of evidence, Princeton university press Princeton, NJ, 1976.

[35] P. Smets, The transferable belief model for quantified belief representation, Handbook of defeasible reasoning and uncertainty management systems 1 (1998) 267-301.

[36] T. Denœux, M. Masson, Evclus: evidential clustering of proximity data, IEEE Transactions on Systems, Man, and Cybernetics, Part B: Cybernetics 34 (1) (2004) 95-109.

[37] J. Sammon, A nonlinear mapping for data structure analysis, IEEE Transactions on computers 18 (5) (1969) 401-409.

[38] M. Masson, T. Denœux, ECM: An evidential version of the fuzzy c-means algorithm, Pattern Recognition 41 (4) (2008) 1384-1397.

[39] W. Wang, Y. Zhang, On fuzzy cluster validity indices, Fuzzy Sets Syst. 158 (19) (2007) 2095-2117.

[40] C. A. Cocosco, V. Kollokian, R. K. S. Kwan, G. B. Pike, A. C. Evans, Brainweb: Online interface to a 3d MRI simulated brain database, NeuroImage 5 (1997) 425 .

[41] R. K. Kwan, A. C. Evans, G. B. Pike, MRI simulation based evaluation of image-processing and classification methods., IEEE Trans Med Imaging 18 (11) (1999) 1085-1097.

[42] R. Kwan, A. C. Evans, G. B. Pike, An extensible MRI simulator for post-processing evaluation, Springer-Verlag, 1996, pp. 135-140

[43] D. L. Collins, A. P. Zijdenbos, V. Kollokian, J. G. Sled, N. J. Kabani, C. J. Holmes, A. C. Evans, Design and construction of a realistic digital brain phantom, IEEE Trans. Med. Imaging (3) (1998) 463-468. 